\title{
0038-1098(95)00070-4
}

\section{AN EXPERIMENTALISTS VIEW ON THE ANALOGY BETWEEN STEP EDGES AND QUANTUM MECHANICAL PARTICLES}

\author{
H.J.W. Zandvliet \\ Faculty of Applied Physics, University of Twente, P.O. Box 217, 7500 AE Enschede, The Netherlands \\ (Received 15 November 1994 by D. Van Dyck)

\begin{abstract}
Guided by scanning tunnelling microscopy images of regularly stepped surfaces it will be illustrated that there is a striking similarity between the behaviour of monoatomic step edges and quantum mechanical particles (spinless fermions). The direction along the step edge is equivalent to the time, while the direction perpendicular to the step corresponds to the one-dimensional space along which the quantum mechanical particles are located. The non-crossing condition of the steps, giving rise to entropic repulsion, is related to the boundary condition of the wavefunction of a quantum mechanical particle at an infinitely high barrier. Finally, the step edge stiffness can be related to
\end{abstract} \\ the mass of the quantum mechanical particle.
}

Keywords: A. semiconductors, C. crystal structure and symmetry, C. scanning tunnelling microscopy.

THE STRUCTURE of vicinal surfaces, i.e., regularly stepped surfaces which are generated by a slight miscut with respect to a low-index plane, has attracted the attention of surface scientists for several decades [1-3]. This interest is not only due to the key role that step edges play in surface phenomena as epitaxial growth, roughening, faceting and etching [1-3] but also due to the strong similarity with the problem of the fluctuation-induced meandering of domain walls [4]. With the advent of scanning tunnelling microscopy (STM) rapid progress in this field of interest has been made due to the ability to observe the step wandering on an atomic scale. In principle, a statistical analysis of such STM images provides a method for determining quantitative values for the important energetic parameters, like e.g., kink formation energies $[5,6]$ and step-step interactions [7-9], which govern the step morphology.

From minimum energy considerations it is evident that at zero temperature a step edge [see Fig. 1a] should be as straight as possible. The only kinks that can occur in a step edge at $T=0 \mathrm{~K}$ arise from an azimuthal misalignment of the step edge with respect to a high symmetry direction. These kinks are called forced kinks and should be discriminated from the thermally excited kinks which emerge if the temperature increases. Under the influence of the thermal generation of additional kinks the step edges start to meander across the surface and will undergo entropy-reducing collisions with neighbouring step edges due to the inability to cross neighbouring step edges [1-4] (step crossings will lead to overhangs which have never been observed experimentally). Let us consider first the very simple case of a meandering step edge trapped between two hard walls, located at $\pm L$. This model seems to be a particularly appropriate model to describe vicinal $\mathrm{Si}(001)$ and $\mathrm{Ge}(001)$ surfaces slightly misoriented towards [1 10 ], where rough and straight monoatomic step edges alternate due to the symmetry of the diamond-type lattice [see Fig. Ib]. The wandering rough step edge has in this particular case the largest configurational entropy when it is midway between the neighbouring straight step edges, leading to a peaked position probability function of the terrace width, $P(h, L)$, at low temperature $[1,2]$

$P(h, L)=\left(\frac{1}{L}\right) \cos ^{2}\left(\frac{\pi h}{2 L}\right)$.

More than two decades ago Gruber and Mullins [1] derived this probability distribution function by evaluating the partition sum of a wandering step 

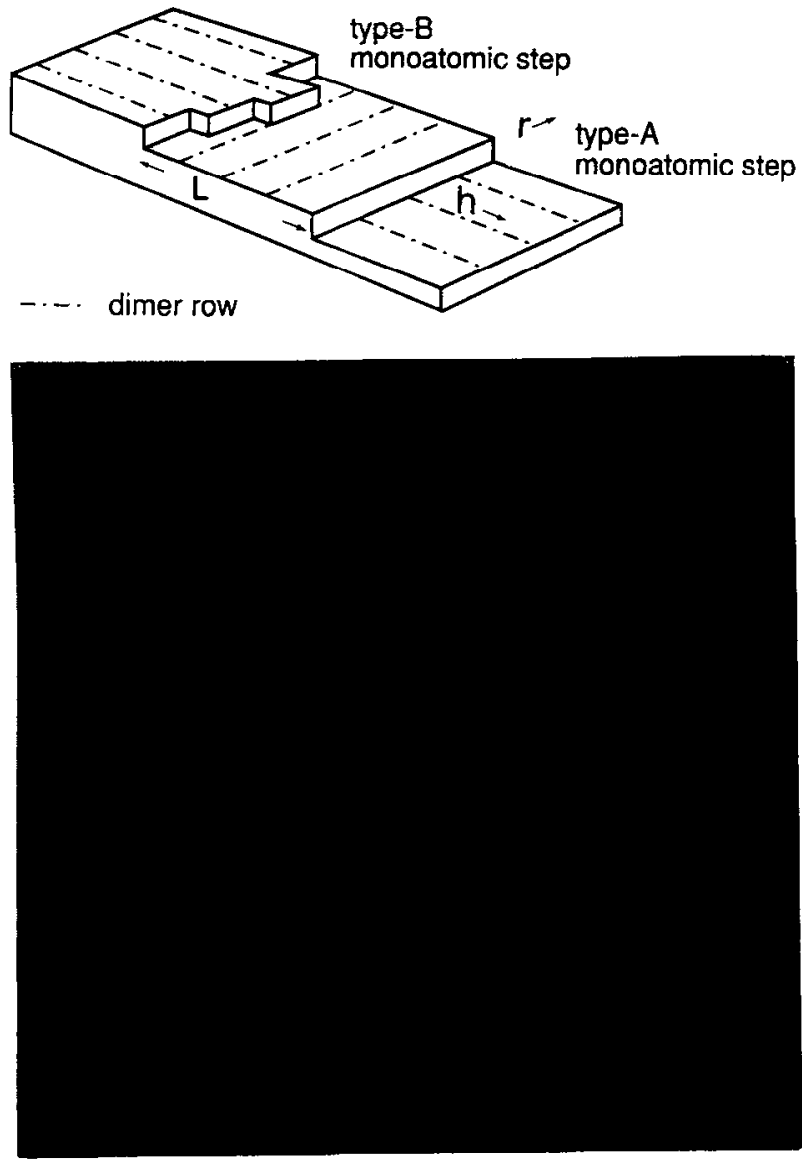

Fig. 1. Schematic diagram (a) and Scanning Tunnelling Microscopy image (b) of a vicinal Si( 001 ) surface containing monoatomic step edges. The tunnelling current was $0.5 \mathrm{nA}$, the sample bias $-2 \mathrm{~V}$ and the scan size $1000 \AA \times 1000 \AA$. The Si $(00$ l) surface reconstructs by forming surface dimers which are arranged in parallel rows. $L$ is the averaged terrace width, $r$ is measured along the step edge and $h$ perpendicular to the step edge. $L, r$ and $h$ are all measured in units of the dimer row-dimer row spacing, i.e. $7.7 \AA$.

edge trapped between two hard walls. It is remarkable that this, in a classical framework obtained, probability distribution function $P(h, L)$ is exactly the same as the probability distribution function of a quantum mechanical particle in a one-dimensional box $[P(h, L)$ can be computed from the square of the wavefunction: $\left.\psi^{*}(h) \psi(h)\right]$. One can understand this similarity if one realizes that the non-crossing condition, which is responsible for what is usually denoted as entropic repulsion, can be related to the boundary conditions of the wavefunction, $\psi$, at the hard wall positions: $\psi(-L)=\psi(L)=0$ in the quantum mechanical problem. Furthermore, the direction along the step edges ( $r$-axis) acts as the time like axis and the step edge stiffness $\gamma\left(\gamma \sim k_{b} T /\left\langle k^{2}\right\rangle\right.$ and $\left\langle k^{2}\right\rangle$ is the mean square displacement of the step edge)
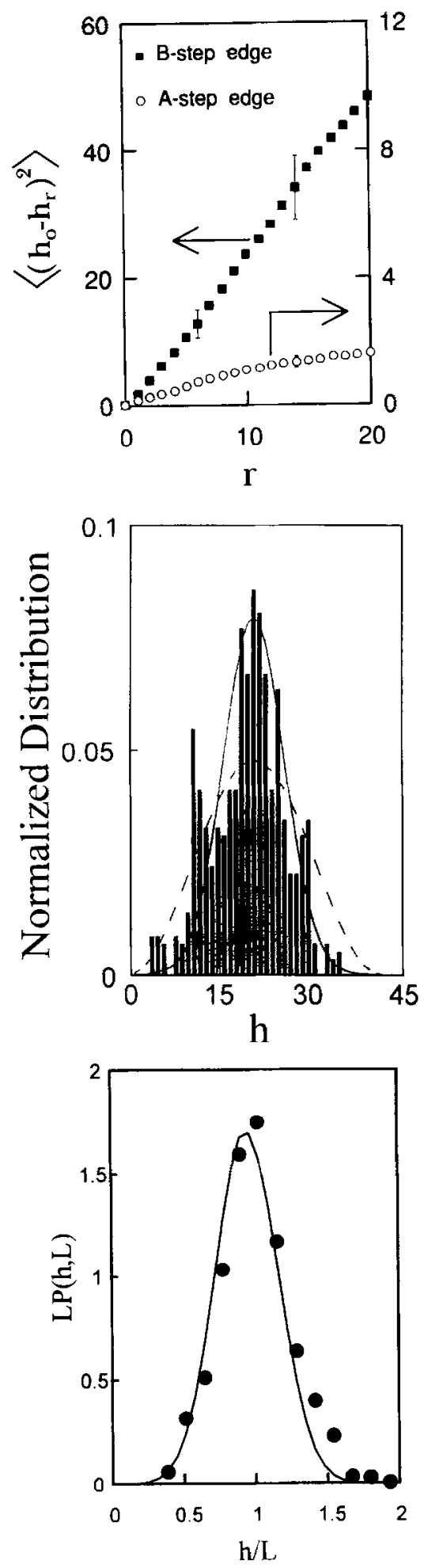

Fig. 2. (a) Mean squares displacement, $\left\langle\left(h_{0}-h_{r}\right)^{2}\right\rangle$, of a straight (A) and a rough (B) monoatomic step edge vs the position difference, $r$, measured along the step edge. Both $h$ and $r$ are measured in units of the dimer row-dimer row spacing, i.e. $7.7 \AA$. Onedimensional random walk behaviour is observed for continued on p. 679. 
corresponds to the mass of the quantum mechanical particle $[2,3,10]$.

In Fig. 2a,b the height-height correlation function, $\left\langle\left(h_{0}-h_{r}\right)^{2}\right\rangle$, of a straight (A-type) and rough (B-type) monoatomic step edge and the probability distribution function of the terrace width, $P(h, L)$, of vicinal $\mathrm{Si}\left(\begin{array}{ll}0 & 0 \\ 1\end{array}\right)$ as obtained from several STM images are shown [7, 9]. Compared to the meandering B-type monoatomic step edge of Si(001) the A-type step edge is nearly straight. In agreement with the evolution of the position-position correlation function, $\left\langle\left(x_{t}-x_{0}\right)^{2}\right\rangle$, of a quantum mechanical particle as a function of time one also finds in the corresponding step edge problem one-dimensional random walk behaviour along the step edge direction, i.e. $\left\langle\left(h_{0}-h_{r}\right)^{2}\right\rangle \sim r$. The probability distribution function of the terrace width as depicted in Fig. $2 b$ is not exactly a cosine squared distribution but a more peaked one indicating that besides the entropic stepstep interaction also a repulsive energetic step-step interaction term must be present. If one includes an energetic step-step interaction term of the form $C L^{-2} h^{2}$ a Gaussian distribution (harmonic oscillator) will be found [3]

$P(h, L)=\frac{1}{w \sqrt{2 \pi}} \mathrm{e}^{-h^{2} / 2 w^{2}}$,

where

$w(T, L)=\left(\frac{k_{b} T\left\langle k^{2}\right\rangle L^{2}}{8 C}\right)^{1 / 4}$.

$k_{b}$ is the Boltzmann constant, $T$ the temperature, and $\left\langle k^{2}\right\rangle$ the mean square displacement of the step edge $\left(\left\langle k^{2}\right\rangle=\Sigma k^{2} n_{k}\right.$, where $k$ is the kink length measured perpendicular to the step edge and $n_{k}$ the probability of finding a kink with length $k$ ). Using the above

both types of monoatomic step edges. (b) Probability distribution function, $P(h, L)$, of the terrace width of a monoatomic stepped Si(001) surface. The average terrace length corresponds to about 20 dimer rowdimer row spacings $\left[0.5^{\circ}\right.$ misoriented $\left.\mathrm{Si}(001)\right]$. Dotted line: probability distribution of a wandering step edge trapped between two fixed walls without any energetic step-step interactions $[P(h L)=$ $\left.(1 / L) \cos ^{2}(\pi h / 2 L)\right]$. Bold line: probability distribution function of a step edge trapped between two fixed walls with energetic step-step interaction $\left[P(h, L)=1 / w \sqrt{2 \pi} \mathrm{e}^{-h^{2} / 2 w^{2}}\right.$ with $\left.w=5\right]$. (c) Modified probability distribution, $L P(h, L)$ vs $h / L$ of a single domain $\mathrm{Si}(001)$ surface containing biatomic step edges. Solid line: a Gaussian. The experimental data set (circles) displays a slightly distorted Gaussianlike behaviour in agreement with [10]. mentioned framework it is even possible to extract quantitative values for the energetic, the entropic step-step interactions and step edge stiffness from the measured experimental curves [5-9].

One can go beyond the approach of one wandering step edge and let all the steps wander simultaneously [10] (this problem is equivalent to that of the domain walls of a striped incommensurate $2 \mathrm{D}$ phase). In fact we now have to solve a many-body quantummechanical problem where each step edge (or domain wall) is replaced by a spinless fermion. Joós and co-workers [10] have extensively analyzed this problem and they found that the probability distribution function of the terrace width, $P(h, L)$, multiplied by $L$, i.e. $L P(h, L)$, plotted vs $h / L$ is a universal curve independent of $L$. In the presence of step--step interactions of the form $L^{-2}$ a slightly distorted Gaussian with an algebraic rise $(h / L)^{4}$ and a Gaussian tail is found whereas in the absence of these interactions a distorted cosine squared distribution with a parabolic rise and a Gaussian tail will be found. In Fig. $2 c$ the distorted Gaussian-like probability distribution function of a single domain $\mathrm{Si}(001)$ surface containing equivalent biatomic step edges as extracted from many STM images is depicted.

In summary, the classical thermodynamic description of step edges on vicinal surfaces can be replaced by a quantum mechanical one in which each step edge is replaced by a spinless fermion. Both approaches, i.e. quantum mechanical and classic thermodynamical, yield in principle the same results because they are both continuum theories that treat the step edge as a continuous function of space. Three clear examples of the quantum mechanical like behaviour of steps are given in this letter: the terrace width distribution, the one-dimensional random walk behaviour of a wandering step edge trapped between two fixed walls and the terrace width distribution function of a train of interacting equivalent step edges.

Acknowledgement-The author thanks Dr H.B. Elswijk from Philips Research Laboratories for continuous support during the course of this work.

\section{REFERENCES}

1. E.E. Gruber \& W.W. Mullins, J. Phys. Chem. Solids 28, 875 (1967).

2. E.D. Williams \& N.C. Bartelt, Science 251, 393 (1991).

3. N.C. Bartelt, T.L. Einstein \& E.D. Williams, Surf. Sci. Lett. 240, L591 (1990).

4. M.E. Fisher \& D.S. Fisher, Phys. Rev. B25, 3192 (1982).

5. B.S. Swartzentruber et al., Phys. Rev. Lett. 65, 1913 (1990). 
6. H.J.W. Zandvliet et al., Phys. Rev. B45, 5965 (1992).

7. H.J.W. Zandvliet et al., Phys. Rev. Lett. 70, 2122 (1993).

8. B.S. Swartzentruber et al., Phys. Rev. B47, 13432 (1993).
9. H.J.W. Zandvliet \& H.B. Elswijk, Phys. Rev. B48, 14269 (1993).

10. B. Joós, T.L. Einstein \& N.C. Bartelt, Phys. Rev. B43, 8153 (1991). 